EPJ Web of Conferences 32, 01002 (2012)

DOI: $10.1051 /$ epjconf/20123201002

(C) Owned by the authors, published by EDP Sciences, 2012

\title{
Anomalous phenomena in ECRH experiments at toroidal devices and low-threshold parametric decay instabilities
}

\author{
E.Z. Gusakov ${ }^{1,2}$, A.Yu. Popov ${ }^{1,2}$, A.N. Saveliev ${ }^{1,2}$ \\ ${ }^{1}$ Ioffe Institute of RAS, 26 Polytekhnicheskaya st., St.-Petersburg 194021, Russia \\ ${ }^{2}$ RLPAT, St. Petersburg State Polytechnical University, 29 Polytekhnicheskaya st., St .Petersburg \\ 195251, Russia
}

\begin{abstract}
In the paper the possibility of total 3D trapping of electron Bernstein (EB) waves in the tokamak equatorial plane in the vicinity of the local density maximum produced by electron pump-out-effect is demonstrated. Thresholds and growth rates of the associated absolute (temporally growing) parametric decay instability (PDI) leading to anomalous absorption is predicted in the range of less than $100 \mathrm{~kW}$. Its possible role in explanation of ion acceleration observed in ECRH experiments as well as in redistribution of the deposited power is discussed.
\end{abstract}

\section{Introduction}

Electron cyclotron resonance heating (ECRH) at power level of up to $1 \mathrm{MW}$ in a single microwave beam is routinely used in present day tokamak and stellarator experiments and planed for application in ITER for plasma heating, current drive and neoclassical tearing mode island control. Parametric decay instabilities (PDI) leading to anomalous reflection and/or absorption of microwave power are believed to be deeply suppressed in tokamak megawatt power level electron cyclotron (EC) resonance fundamental harmonic ordinary mode and $2^{\text {nd }}$ harmonic extraordinary mode heating experiments utilizing gyrotrons [1] - [3]. Therefore the wave propagation and absorption in these experiments are thought to be well described by linear theory and thus predictable in detail.

However during the last decade a number of observations have been obtained evidencing presence of anomalous phenomena that accompany ECRH experiments at toroidal devices.

First of all, non local electron transport was shown to accompany ECRH in some cases indicating that the RF power is not deposited in the regions predicted by standard theory, but is rather redistributed very quickly all over the plasma [4].

Secondly, the first observations of the backscattering signal in the $200-600 \mathrm{~kW}$ level second harmonic ECRH experiment at Textor tokamak were reported [5], [6], which can be explained in terms of an anomalous backscattering of the EC pump waves.

And finally, fast ion generation was observed during ECRH pulse under conditions when energy exchange between electrons and ions should be very low [7], [8].

It is worth noting here that the later two phenomena were observed at the non-monotonic plasma density profile, caused in each specific case by different physical mechanisms such as features of 
plasma confinement in the magnetic island or so-called electron pump-out effect, originated due to the anomalous convective particle fluxes from the EC layer at the intensive ECRH.

The novel low threshold mechanism of the PDI excitation was proposed in [9] based on analysis of the actual Textor density profile. It was shown that the local maximum of the plasma density, which is usually observed in the O-point of magnetic island at Textor [10], can lead to localisation of the low frequency ion Bernstein (IB) decay wave and thus to suppression of IB wave convective losses in radial direction. A more complicated 2D analysis of the IB wave propagation accounting for the poloidal inhomogenuity of magnetic field in toroidal plasma have shown possibility of IB wave localization in the poloidal direction, as well [11], [12]. The threshold of the backscattering PDI was calculated in this case and shown to be more than four orders of magnitude lower than predictions of standard theory (in the range of $50 \mathrm{~kW}$ for the Textor experiment parameters).

Quite recently, the possibility of the IB wave 3D localization and the low-threshold absolute PDI excitation was reported in [13]. The analysis performed at fusion relevant parameters for the planned ECRH experiments in JET predicts the threshold of the absolute PDI in the range of $100 \mathrm{~kW}$ being approximately four orders of magnitude lower than that predicted by the standard theory [1] - [3] and an order of magnitude lower than the threshold of the fast convective PDI. Nevertheless, at low plasma density and temperature the growth rate of this instability appears to be too small to make the instability important for the energy budget.

It should be stressed however, that at these plasma parameters another scenario of the lowthreshold absolute PDI of the extraordinary mode EC wave, leading to its anomalous absorption via decay into low frequency IB wave and high frequency electron Bernstein (EB) wave, may be realized at the non-monotonic density profile as well. The EB wave in this case is trapped in the equatorial plane near the density maximum in a $3 \mathrm{D}$ toroidal cavity that substantially decreases the threshold of instability, whereas the IB wave propagates to the nearest ion cyclotron harmonic layer and accelerates ions.

In the present paper the experimental conditions leading to the 3D EB wave trapping and substantial reduction of the threshold of the anomalous absorption in ECRH experiments are analyzed. The absolute PDI excitation is predicted and the corresponding threshold is shown to be much smaller than that provided by the standard theory [1-3].

\section{Theoretical approaches}

To elucidate the physics of the absolute PDI we analyze the most simple but nevertheless relevant to the experiment [8] three wave interaction model in which the extraordinary mode pump wave propagates almost perpendicular to the magnetic field $\vec{H}$ in the density inhomogeneity direction $x$ with its polarization vector being mostly directed along the poloidal direction $y$. We represent a wide microwave beam of the extraordinary mode pump wave propagating from the launching antenna inwards plasma along major radius in the tokamak mid-plane as

$$
E_{0 y}=a_{0}(y, z) / 2 \cdot \exp \left(q_{0} x-i \omega_{0} t\right)+\text { c.c. }
$$

where c.c. is complex conjugation, $z$ stands for toroidal direction being periodic in tokamak, $a_{0}=\sqrt{8 \pi / c \cdot P_{0} /\left(\pi w^{2}\right)} \exp \left[-\left(y^{2}+z^{2}\right) /\left(2 w^{2}\right)\right]$ is the amplitude, $P_{0}$ is the pump wave power and $w$ is the beam waist. The basic set of integral-differential equations describing the decay of the extraordinary mode pumping wave (1) into a daughter IB wave and EB wave: $\vec{E}_{I}=-\vec{\nabla} \phi_{I} \exp (i \Omega t)$, $\vec{E}_{E}=-\vec{\nabla} \phi_{E} \exp \left(-i\left(\omega_{0}-\Omega\right) t\right)$, where $\Omega<<\omega_{0}$, is given as:

$$
\left\{\begin{array}{l}
\hat{D}\left(\vec{r}, \vec{r}^{\prime} ; \Omega\right)\left\{\phi_{I}\left(\vec{r}^{\prime}\right)\right\}=4 \pi \rho_{I}(\vec{r} ; \Omega) \\
\hat{D}\left(\vec{r}, \vec{r}^{\prime} ; \omega_{0}-\Omega\right)\left\{\phi_{E}\left(\vec{r}^{\prime}\right)\right\}=4 \pi \rho_{E}\left(\vec{r} ; \omega_{0}-\Omega\right)
\end{array}\right.
$$

The integral operators $\hat{D}$ in (2) are defined in weakly inhomogeneous plasma as follows: 


$$
\hat{D}\left(\vec{r}, \vec{r}^{\prime} ; \omega\right)\left\{f\left(\vec{r}^{\prime}\right)\right\}=\int_{-\infty}^{\infty} \frac{d \vec{q} d \vec{r}^{\prime}}{(2 \pi)^{3}} D\left(\vec{q}, \frac{\vec{r}+\vec{r}^{\prime}}{2} ; \omega\right) \exp \left[i \vec{q}\left(\vec{r}-\vec{r}^{\prime}\right)\right] f\left(\vec{r}^{\prime}\right),
$$

where $\omega=\left[\Omega, \omega_{0}-\Omega\right], D(\vec{q}, \vec{r} ; \omega)=q^{2}+\chi_{e}(\vec{q}, \vec{r} ; \omega)+\chi_{i}(\vec{q}, \vec{r} ; \omega), \quad \chi_{e}$ and $\chi_{i}$ being defined at a fixed coordinate $\vec{r}$ and consisting of the real and imaginary part are familiar expressions for electron and ion susceptibilities in homogeneous plasmas [14], [15]:

$$
\begin{aligned}
& \chi_{e}\left(\omega_{0}-\Omega\right)=\frac{2 \omega_{p e}^{2}}{v_{t e}^{2}}\left[1-\sum_{m=-\infty}^{\infty} \frac{\omega_{0}-\Omega}{q_{\|} v_{t e}} Z\left(\frac{\omega_{0}-\Omega-m \omega_{c e}}{q_{\|} v_{t e}}\right) \exp \left(-\frac{q_{\perp}^{2} v_{t e}^{2}}{2 \omega_{c e}^{2}}\right) I_{m}\left(\frac{q_{\perp}^{2} v_{t e}^{2}}{2 \omega_{c e}^{2}}\right)\right]+i q_{\perp}^{2} \frac{\omega_{p e}^{2}}{\omega_{c e}^{3}} v_{e i} ; \\
& \chi_{e}(\Omega)=\frac{2 \omega_{p e}^{2}}{v_{t e}^{2}}\left[1-\frac{\omega_{0}-\Omega}{q_{\|} v_{t e}} Z\left(\frac{\Omega}{q_{\|} v_{t e}}\right) \exp \left(-\frac{q_{\perp}^{2} v_{t e}^{2}}{2 \omega_{c e}^{2}}\right) I_{0}\left(\frac{q_{\perp}^{2} v_{t e}^{2}}{2 \omega_{c e}^{2}}\right)\right]-i q_{\perp}^{2} \frac{\omega_{p e}^{2}}{\omega_{c e}^{2}} \frac{v_{e i}}{\Omega} \\
& \chi_{i}(\Omega)=\frac{2 \omega_{p i}^{2}}{v_{t i}^{2}}\left[1-X\left(\frac{\Omega}{q_{\perp} v_{t i}}\right)-Y\left(\frac{\Omega}{q_{\perp} v_{t i}}\right)\left\{\cot \left(\pi \frac{\Omega}{\omega_{c i}}\right)+\frac{i}{\sqrt{\pi}} \frac{\omega_{c i}}{q_{\|} \mid v_{t i}} \sum_{m=-\infty}^{\infty} \exp \left(-\frac{\left(\Omega-m \omega_{c i}\right)^{2}}{q_{\|}^{2} v_{t i}^{2}}\right)\right\} ;\right.
\end{aligned}
$$

where $\quad q_{\perp}=|\vec{q} \times \vec{H}| /|H|, \quad q_{\|}=\vec{q} \cdot \vec{H} /|H| \quad$ are components of the wave vector, $\vec{H}=|H|\left(\sin \alpha \cdot \vec{e}_{y}+\cos \alpha \cdot \vec{e}_{z}\right)$ is an external magnetic field composed of the toroidal and poloidal components, $\sin \alpha=H_{y} /|H|<<1, \quad I_{m}$ is modified Bessel function of the first kind; and $\chi_{i}^{\prime}\left(\omega_{0}-\Omega\right)<<\chi_{e}^{\prime}\left(\omega_{0}-\Omega\right) ; \xi Z(\xi)=X(\xi)-i Y(\xi)=\frac{\xi}{\sqrt{\pi}} \int_{-\infty}^{\infty} \exp \left(-t^{2}\right) \frac{d t}{\xi-t}$.

We have also introduced in (2) the nonlinear charge densities $\rho_{I}(\Omega)$ and $\rho_{E}\left(\omega_{0}-\Omega\right)$ generated by the ponderomotive force and being responsible for coupling of low and high frequency waves [16]:

$$
\rho_{I}(\Omega) \simeq \chi_{i}(\Omega) \frac{a_{B}^{*}}{8 \pi} \frac{\chi_{e}\left(\omega_{0}-\Omega\right) \phi_{E}}{q_{\perp}^{2}}, \rho_{E}\left(\omega_{0}-\Omega\right) \simeq \chi_{e}\left(\omega_{0}-\Omega\right) \frac{a_{B}}{8 \pi} \frac{\chi_{i}(\Omega) \phi_{I}}{q_{\perp}^{2}},
$$

where $a_{B}=E_{0 y} / H \cdot \omega_{c e}^{2} /\left(\omega_{0}^{2}-\omega_{c e}^{2}\right) \approx E_{0 y} /(3 H)$.

\section{The reduced Bernstein wave equations}

As it was shown in [9], [11-13], the PDI threshold decreases substantially when one of the daughter waves, IB wave in the particular case of these references, is trapped at least in $x$ - direction.

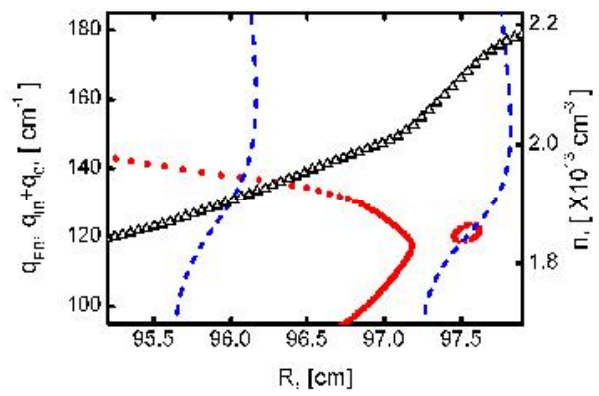

Fig. 1: (Colour on-line) (a, left and bottom axes): EB wave 1D dispersion curves $q_{E B}(x)$ (solid lines) and IB dispersion curves $q_{I B}(x)$ up-shifted by the wave-vector of the pump wave (dashed lines) versus the coordinate $x-x_{m}$ where $x_{m}$ is a position of the density maximum. In the points where $q_{E B}(x)$ and $q_{I B}(x)+q_{0}$ intersect the Bragg resonance conditions are fulfilled; (b, right and bottom axes): plasma density (solid line with triangles) versus the coordinate $x-x_{m}$. 
This is also possible for the EB wave if the turning point of its dispersion curve and the local maximum of the non-monotonous density profile are close one to another. Seeking a solution of the system

$$
\left.\left.D^{\prime}\left(\vec{q}, \omega_{0}-\Omega, x\right)\right|_{\vec{q}_{E}, \omega_{E}, x_{E}} \equiv D^{\prime}\left(\vec{q}, \omega_{0}-\Omega, x\right)\right|_{E}=0 ; \quad \partial D^{\prime}\left(\vec{q}, \omega_{0}-\Omega, x\right) /\left.\partial q_{x}\right|_{E}=0 ; \partial D^{\prime}\left(q, \omega_{0}-\Omega, x\right) /\left.\partial x\right|_{E}=0,
$$

where the first equation is the dispersion relation of the EB wave, the second one is a condition of the turning point of its dispersion curve and the latter is the extremum condition for the dispersion function $\left.D^{\prime}\left(\vec{q}, \omega_{0}-\Omega, x\right)\right|_{E}$ over variable $x$, we can get frequency $\omega_{0}-\Omega=\omega_{E}$, wave number $\vec{q}=\vec{q}_{E}=\left(q_{E}, 0,0\right)$ and coordinate $x=x_{E}$ which, while additional constraints $\partial^{2} D^{\prime}\left(\vec{q}, \omega_{0}-\Omega, x\right) /\left.\partial q_{x}^{2}\right|_{E}>0, \partial^{2} D^{\prime}\left(q, \omega_{0}-\Omega, x\right) /\left.\partial x^{2}\right|_{E} \equiv q_{E}^{2} / L^{2}>0$ hold, provide a global minimum of the dispersion function $D^{\prime}\left(q, \omega_{E}, x\right)$ over two variables $(q, x)$. This guarantees the existence of two nearby turning points ("warm" to "hot" mode) of the EB wave dispersion curve in plasma and EB wave trapping between them leading to entire suppression of the corresponding convective losses. The trapping of the EB wave is shown in Figure 1 where its dispersion curves at frequency $\omega_{E B} / 2 \pi=\left(\omega_{0}-\Omega_{I}\right) / 2 \pi=76.4 \mathrm{GHz}$ together with the density profile are depicted for the typical parameters of TCV ECRH experiments [18] $\left(R_{0}=87.5 \mathrm{~cm}, a=24 \mathrm{~cm}, T_{e}=1.8 \mathrm{keV}, T_{i}=350 \mathrm{eV}\right.$, $H\left(x_{m}\right)=12.6 \mathrm{kGs}, \quad n\left(x_{m}\right)=2.2 \times 10^{13} \mathrm{~cm}^{-3}, \omega_{0} / 2 \pi=77 \mathrm{GHz}$ and $x_{m}$ is a position of the density maximum). This figure also illustrates the possibility of three-wave interaction of the EB wave with the $2^{\text {nd }}$ harmonic extraordinary mode pump wave and the IB wave at frequency $\Omega_{I} / 2 \pi=0.6 \mathrm{GHz}$. The dispersion curve for the latter is shown in Figure 1 up-shifted by the pump wave number $q_{0}=17.2 \mathrm{~cm}^{-1}$.

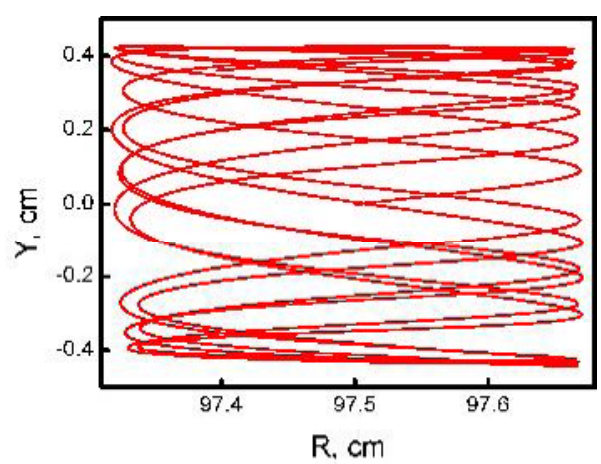

Fig. 2: (Colour on-line) Trajectory of the EB wave in the $q_{E}=118 \mathrm{~cm}^{-1}$ poloidal cross - section. $T_{e}=1.8 \mathrm{keV}, \omega_{E} / 2 \pi=76.4 \mathrm{GHz}$, the background density profile $n(x)$ is chosen as in fig. 1.

The poloidal dependence of the magnitude of the magnetic field can ensure the localization of the EB wave also in poloidal direction. As it is shown in Figure 2 by the results of the EB wave ray tracing analysis performed accounting for the tokamak equilibria for the same parameters and profiles as used in Figure 1, the ray trajectory is localised in the finite plasma volume. The phase portraits of the motion in radial and poloidal direction are represented by elliptic curves shown in Figure 3 that corresponds to the finite motion. Accordingly, in a vicinity of the turning point of the EB wave $q_{x}=q_{E}$, the local maximum of the dispersion function in the radial direction $x=x_{E}$ (further we will assume $x_{E}=0$ ) and the minimum of the magnetic field in the poloidal direction $y=0$ the system of integral-differential equation (2) with (3) and (4) reduces to 


$$
\begin{gathered}
\left\{i D_{I q_{x}}^{\prime} \frac{\partial}{\partial x}-\frac{\omega_{p e}^{2}}{\Omega_{I}^{2}} \frac{\partial^{2}}{\partial \xi^{2}}+i D_{I}^{\prime \prime}\right\} b_{I}=\exp \left[-i \int^{x} \Delta q d x^{\prime}\right] \frac{\left|a_{B}\right|}{2} \frac{\chi_{i}\left(\Omega_{I}\right) \chi_{e}\left(\omega_{E}\right)}{q_{E}^{2}} b_{E} \\
\left\{\hat{D}_{E}+i D_{E}^{\prime \prime}\right\} b_{E}=\exp \left[i \int^{x} \Delta q d x^{\prime}\right] \frac{\left|a_{B}\right|}{2} \frac{\chi_{e}\left(\omega_{E}\right) \chi_{i}\left(\Omega_{I}\right)}{q_{I}^{2}} b_{I}
\end{gathered}
$$

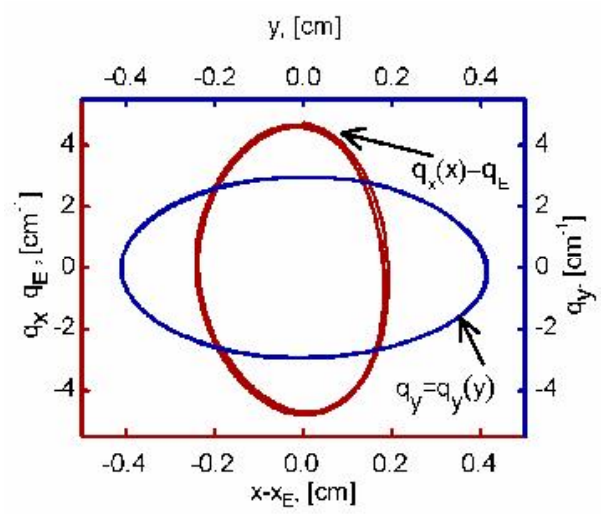

Fig. 3: (Colour on-line) (a, left and bottom axes): The phase portrait $q_{x}(x)-q_{E} ;$ (b, right and top axes): The phase portrait $q_{y}(y)$; The parameters are chosen as in Figure 2.

where $b_{I, E}(\vec{r})$ are slowly varying amplitudes of the potentials $\phi_{E, I}(\vec{r})$ defined as $\phi_{E}(\vec{r})=b_{E}(\vec{r}) \exp \left(i q_{E} x-i \omega_{E} t\right), \quad \phi_{I}(\vec{r})=b_{I}(\vec{r}) \exp \left(i \int^{x} q_{I} d x+i \Omega_{I} t\right) ; \vec{q}_{I}=\left(q_{I}(x), 0,0\right)$ is a solution of the IB wave dispersion relation $D^{\prime}\left(\vec{q}_{I}, \Omega_{I}, x\right)=0$ and $\Omega_{I}=\omega_{0}-\omega_{E B} ; \quad D_{I q_{x}}^{\prime}=\partial D^{\prime}\left(q_{I}, \Omega_{I}, x_{E}\right) / \partial q_{x}$; $D_{I}^{\prime \prime}=D^{\prime \prime}\left(\vec{q}_{I}, \Omega_{I}, x_{E}\right) ; \quad \Delta q=q_{0}+q_{I}(x)-q_{E}$ is a small decay condition mismatch; $\hat{D}_{E}=\left.\left(\omega_{E B}-\omega_{E}\right) \frac{\partial D^{\prime}}{\partial \omega}\right|_{E}-\left.\frac{\partial^{2} D^{\prime}}{2 \partial q_{x}^{2}}\right|_{E} \frac{\partial^{2}}{\partial x^{2}}+\frac{q_{E}^{2}}{L^{2}} x^{2}+\frac{q_{E}^{2}}{q_{\xi}^{2}} \frac{\partial^{2}}{\partial \xi^{2}}-\frac{q_{E}^{2}}{y_{0}^{2}} y^{2}$ is a reduced differential form of the EB wave integral operator; $\quad D_{E}^{\prime \prime}=D^{\prime \prime}\left(q_{E}, \omega_{E}, x_{E}\right) ; \quad \frac{1}{y_{0}^{2}}=\frac{\omega_{p e}^{2}}{q_{E}^{2} v_{t e}^{2}\left(x_{m}-R_{0}\right) R_{0}} \sum_{m=-\infty}^{\infty} \frac{1}{\varepsilon_{m}^{2}} \exp \left(-\frac{q_{E}^{2} v_{t e}^{2}}{2 \omega_{c e}^{2}}\right) I_{m}\left(\frac{q_{E}^{2} v_{t e}^{2}}{2 \omega_{c e}^{2}}\right)$; $\frac{1}{q_{\xi}^{2}}=\frac{\omega_{p e}^{2}}{q_{E}^{2} \omega_{0}^{2}} \sum_{m=-\infty}^{\infty} \frac{1}{\varepsilon_{m}^{3}} \exp \left(-\frac{q_{E}^{2} v_{t e}^{2}}{2 \omega_{c e}^{2}}\right) I_{m}\left(\frac{q_{E}^{2} v_{t e}^{2}}{2 \omega_{c e}^{2}}\right) ; \quad \varepsilon_{m}(x)=1-m \omega_{c e}(x) /\left(\omega_{0}-\Omega_{0}\right)$ and coordinates along and across magnetic field on the magnetic surface are introduced by relations $y=\cos \alpha \cdot \eta+\sin \alpha \cdot \xi$, $z=-\sin \alpha \cdot \eta+\cos \alpha \cdot \xi$.

\section{Threshold and growth rate of the absolute PDI}

We perform the analysis of the EB wave toroidal cavity parametric excitation using the perturbation theory approach. Following approach of [17], at the first step of the perturbation procedure we assume that the trapped EB wave and IB wave propagating between two nearby IC harmonics are not interacting. Thus assuming the EB wave PDI pumping (r.h.s. of equation (6)) and its damping (see second term in 1.h.s. of equation (6)) small, we neglect them in the zero order approximation and obtain equation which can be solved by separation of variables:

$$
b_{E}^{k n l}(x, y, z)=\exp \left(i \frac{l}{R_{0} \sin \alpha} \eta\right) \varphi_{k}(x) \varphi_{n}(y)
$$


where $\varphi_{k}(x) \varphi_{n}(y)=H_{k}\left(\frac{x}{\delta_{x}}\right) H_{n}\left(\frac{y}{\delta_{y}}\right) \exp \left[-\frac{x^{2}}{2 \delta_{x}^{2}}-\frac{y^{2}}{2 \delta_{y}^{2}}\right], H_{k}$ are Hermitian polinomials, the sizes of the EB mode localization region in the radial and poloidal directions are $\delta_{x}=\sqrt[4]{\partial^{2} D_{E}^{\prime} /\left.\left(2 \partial q_{x}^{2}\right)\right|_{E}} \sqrt{L / q_{E}}$ and $\delta_{y}=\sqrt{\sin \alpha \cdot y_{0} / q_{\xi}}$, accordingly. The exact value of the mode frequency $\omega_{E B}=\omega_{E}+\delta \omega_{k, n}$ is determined by following quantization condition

$$
\delta \omega_{k, n}=\left.\frac{\partial D_{E}^{\prime}}{\partial \omega}\right|_{E} ^{-1}\left[\sin |\alpha| \frac{q_{E}^{2}}{q_{\xi} y_{0}}(2 n+1)-\left.\sqrt{\frac{\partial^{2} D_{E}^{\prime}}{2 \partial q_{x}^{2}}}\right|_{E} \frac{q_{E}}{L}(2 k+1)\right] .
$$

At the next step of the perturbation theory procedure we take the EB wave damping and PDI pumping into account. Assuming the EBW is localized in $x$ direction in a very narrow layer close to $x_{E}=0$ where the IBW damping is weak we neglect the last term $D_{I}^{\prime \prime}$ in the 1.h.s. of equation (5) describing nonlinear excitation of the IB wave, its damping as well as losses both along the magnetic field and in radial direction. We integrate equation (5) to obtain $b_{I}$ in the form

$$
b_{I}=\frac{\chi_{i}\left(\Omega_{I}\right) \chi_{e}\left(\omega_{E}\right)}{2 i q_{E}^{2}} \int_{-\infty}^{x} \frac{d x^{\prime}}{D_{I q_{x}}^{\prime}} \int_{-\infty}^{\infty} d \xi^{\prime} \cdot G\left(x-x^{\prime}, \xi-\xi^{\prime}\right) \exp \left[i \int^{x^{\prime}} \Delta q d x^{\prime \prime}\right]\left|a_{B}\right|\left(y\left(\xi^{\prime}, \eta\right), z\left(\xi^{\prime}, \eta\right)\right) b_{E}\left(x^{\prime}, y\left(\xi^{\prime}, \eta\right), z\left(\xi^{\prime}, \eta\right)\right)
$$

where $G\left(x-x^{\prime}, \xi-\xi^{\prime}\right)=\sqrt{\frac{i\left|D_{I q_{x}}^{\prime}\right| \Omega_{I}^{2}}{4 \pi \omega_{p e}^{2}\left|x-x^{\prime}\right|}} \exp \left[-i \frac{\left|D_{I q_{x}}^{\prime}\right| \Omega_{I}^{2}\left(\xi-\xi^{\prime}\right)^{2}}{4 \omega_{p e}^{2}\left(x-x^{\prime}\right)}\right]$ and the inequalities $q_{0}<<q_{I}, q_{E}$ and $\delta \omega_{k, n}<<\omega_{E}$ were used. Substituting (8) into (6) and using the zero order solution (7) for the EB wave potential in the r.h.s. of (6) we can obtain the first order perturbation theory corrections to the localized EB wave eigen frequency. Namely, multiplying both sides of the equation by the complex conjugated zero order eigen function (7) and performing integration over coordinates we get for the EB eigen mode $(k, n)$ a condition connecting its growth (decay) rate $\gamma$ with the pump wave power:

$$
\begin{aligned}
& \left.\gamma \frac{\partial D_{E}^{\prime}}{\partial \omega}\right|_{E}=-D_{E}^{\prime \prime}+\frac{\chi_{e}^{2}\left(\omega_{E}\right) \chi_{i}^{2}\left(\Omega_{I}\right)}{4 q_{E}^{4} D_{I q_{x}}^{\prime}} \frac{a_{B}^{2}}{2 \pi R_{E}} \frac{1}{\sqrt{\pi} 2^{k} k ! \delta_{x}} \frac{1}{\sqrt{\pi} 2^{n} n ! \delta_{y}} \int_{-\infty}^{\infty} d s \int_{0}^{\infty} d \tau \cdot \varphi_{k}^{*}\left(s+\frac{\tau}{2}\right) \varphi_{k}\left(s-\frac{\tau}{2}\right) \times \\
& \exp \left[-i \int_{s-\tau / 2}^{s+\tau / 2} \Delta q d x\right] \int_{-\infty}^{\infty} d \eta \exp \left(-\frac{\eta^{2}}{w^{2}}\right) \int_{-\infty}^{\infty} d u \int_{-\infty}^{\infty} d v \cdot G(\tau, v) \exp \left(-\frac{u^{2}}{w^{2}}-\frac{v^{2}}{4 w^{2}}\right) \varphi_{n}^{*}\left[y\left(u+\frac{v}{2}, \eta\right)\right] \varphi_{n}\left[y\left(u-\frac{v}{2}, \eta\right)\right]
\end{aligned}
$$

where $R_{E}$ stands for the major radius at $x_{E}=0$. We illustrate the above equation (9) in the most dangerous case of the fundamental EB mode $(k=0, n=0)$ excitation, possessing the minimal PDI threshold. Neglecting for the sake of simplicity the mismatch of decay condition by putting $\Delta q=0$ we obtain for the growth (damping) rate of the PDI an expression

$$
\gamma=-v_{E}+\left.\frac{\partial D_{E}^{\prime}}{\partial \omega}\right|_{E} ^{-1} \frac{\chi_{e}^{2}\left(\omega_{E}\right) \chi_{i}^{2}\left(\Omega_{I}\right)}{4 q_{E}^{4} D_{I q_{x}}^{\prime}} a_{B}^{2} \frac{w^{2} \delta_{x}}{\sqrt{w^{2}+\delta_{y}^{2}}} \frac{J}{2 R_{E}}
$$

where $v_{E}=\left.D^{\prime \prime}\right|_{E} \cdot \partial D^{\prime} /\left.\partial \omega\right|_{E} ^{-1}$ is the EB wave damping rate,

$$
J=\frac{\operatorname{Re}}{\sqrt{\pi}}\left\{\int_{0}^{\infty} \frac{d \xi}{\sqrt{1-i T \xi}} \exp \left[-\frac{\xi^{2}}{4}\right]\right\}
$$

and $T=\tau_{\perp} / \tau_{\|}$is a coefficient equal to ratio of wave energy convective losses time in radial direction, i.e. $\tau_{\perp}=\delta_{x} D_{I \omega}^{\prime} / D_{I q_{x}}^{\prime}$, and diffractive loss time along the magnetic field, i.e. $\tau_{\|}=\Omega^{2} / \omega_{p e}^{2} \cdot D_{I \omega}^{\prime} w^{2}$. In the case $T<<1$ when the convective losses of the IB wave in the radial direction dominate the integration in (11) yields $J=1$. In the opposite case $T \gg>1$ corresponding to a narrow pump beam the diffractive losses of the IB wave along the magnetic field suppress the efficiency of the 
parametric decay by the factor $\operatorname{Re}(J)=\Gamma(1 / 4) /(2 \sqrt{\pi T})$. Assuming in (10) the growth rate equal to zero $\gamma=0$ yields in the latter case the PDI threshold:

$$
\left.\frac{E_{0}^{2}}{H^{2}}\right|_{t h}=v_{E} \frac{\partial D_{E}^{\prime}}{\partial \omega} \frac{36 q_{E}^{4} D_{I q_{x}}^{\prime}}{\chi_{e}^{2}\left(\omega_{E}\right) \chi_{i}^{2}\left(\Omega_{I}\right)} \frac{\sqrt{w^{2}+\delta_{y}^{2}}}{w^{2}} \frac{2 R_{E}}{\delta_{x}} \frac{2 \sqrt{\pi T}}{\Gamma(1 / 4)}
$$

This lengthy expression can be reformulated in the compact form resembling the homogeneous plasma PDI theory result

$$
\left.\gamma_{0}^{2}\right|_{\text {th }} \simeq v_{E} v_{I}^{*} \frac{V_{\text {damp }}}{V_{\mathrm{PDI}}}
$$

Here $\gamma_{0}^{2}=\left|a_{B}\right|^{2} / 4 \cdot \chi_{e}^{2}\left(\omega_{E}\right) \chi_{i}^{2}\left(\Omega_{I}\right) /\left(q_{E}^{4} D_{I \omega}^{\prime} D_{E \omega}^{\prime}\right)$ is the PDI growth rate in homogeneous plasma theory neglecting the daughter wave damping; $v_{I}^{*}=\left(\tau_{\perp} \tau_{\|}\right)^{-1 / 2}$ characterize convective IB wave losses from the $3 \mathrm{D}$ region where three-wave interaction takes place and $V_{\mathrm{PDI}} / V_{\text {damp }}$ is a geometrical factor determined by ratio of two volume elements within which the PDI occurs ( $V_{\mathrm{PDI}} \simeq \pi w^{2} \delta_{y} \delta_{x} / \sqrt{w^{2}+\delta_{y}^{2}}$ ) and the EC resonance damping is significant $\left(V_{\text {damp }}\right)$. In the case of collisional damping of the EB wave $V_{\text {damp }} \simeq 2 \pi R_{E} \delta_{y} \delta_{x}$ and $V_{\mathrm{PDI}} / V_{\text {damp }} \simeq w^{2} /\left(2 R_{0} \sqrt{w^{2}+\delta_{y}^{2}}\right)$. Dependence of the absolute PDI growth rate on the pump power is shown in Figure 4 for parameters of TCV typical for ECRH experiments [18] $\left(R_{0}=87.5 \mathrm{~cm}, a=24 \mathrm{~cm}, T_{e}=1.8 \mathrm{keV}, T_{i}=350 \mathrm{eV}, \quad H\left(x_{m}\right)=12.6 \mathrm{kGs}, \quad n\left(x_{m}\right)=2.2 \times 10^{13} \mathrm{~cm}^{-3}\right.$, $\left.w=2 \mathrm{~cm}, \omega_{0} / 2 \pi=77 \mathrm{GHz}\right)$ resulting in the following PDI characteristics $\left(q_{E}=118 \mathrm{~cm}^{-1}\right.$, $\delta_{x}=0.184 \mathrm{~cm}, \quad \delta_{y}=0.34 \mathrm{~cm}, \quad \Omega_{I} / 2 \pi=0.6 \mathrm{GHz}, \quad \omega_{E B} / 2 \pi=77 \mathrm{GHz}, \quad \omega_{E} / 2 \pi=76.4 \mathrm{GHz}$, $\left.\delta \omega_{0,0} / 2 \pi=-38 \mathrm{MHz}\right)$.

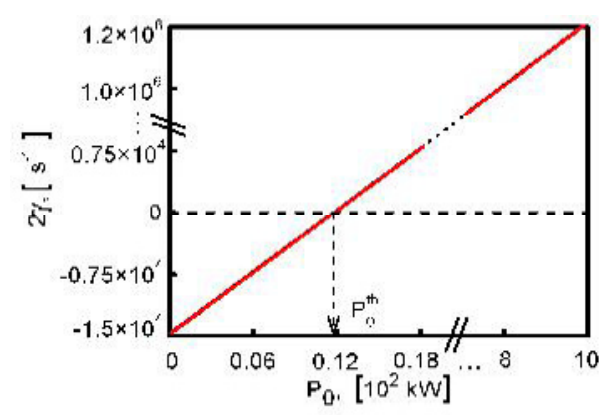

Fig. 4: (Colour on-line) Growth rate of PDI $2 \gamma$ versus power $P_{0}$ of the extraordinary mode EC wave for fundamental EB mode.

As is seen, the instability threshold is only $12 \mathrm{~kW}$ whereas the growth rate at the power level of 400 $600 \mathrm{~kW}$ usually used in tokamak ECRH experiments is high enough $\left(\gamma \simeq 10^{5} s^{-1}\right)$ to complicate quasi linear instability saturation by variation of plasma parameters due to MHD or transport phenomena. Dependence of the PDI threshold pump power on the microwave beam waist obtained from equation (10) and shown in Figure 5 deserves a few comments. At the small beam waist the inequality $1<<T$ holds so that the IB wave diffraction losses along the magnetic field line dominate over its radial convective losses, moreover the EB wave poloidal localization region is larger than the PDI pump region when $w \leq \delta_{y}$. As is shown in Figure 5, in this limit the PDI threshold is increasing $P_{0}^{t h} \propto 1 / w$ as $w$ decreases. In the opposite case when the beam waist is large so that the PDI pump region is 


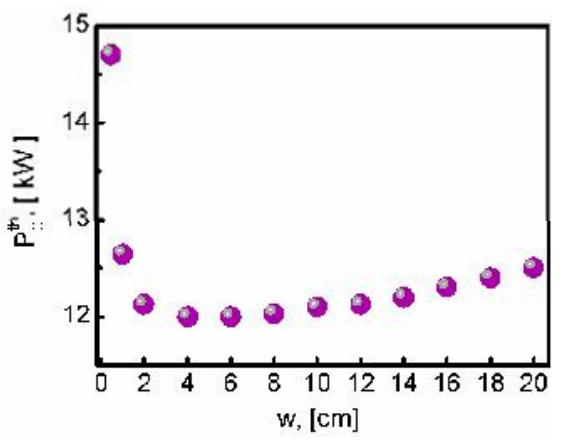

Fig. 5: (Colour on-line) PDI threshold versus the microwave beam waist for the parameters used in Figure 4.

wider than the EB wave localization domain $w \gg>\delta_{y}$ and moreover the radial losses of the IB wave dominate over those along the magnetic field, i.e. $T<<1$, the PDI threshold is increasing $P_{0}^{\text {th }} \propto w$. In the intermediate range the dependence possesses minimum as can be seen in Figure 5 . The minimal threshold value is more than order of magnitude smaller than that calculated for the induced backscattering absolute PDI [13] for the same TCV parameters. The growth rate is an order of magnitude higher than in the later case, which makes the $2^{\text {nd }}$ harmonic extraordinary mode decay into electron and ion Bernstein waves a main candidate for explanation of fast ion acceleration observed in experiment [8]. This mechanism, leading to the microwave power absorption far from the actual ECR layer position, can be to some extent responsible for the non-local electron transport effect often observed in the ECRH experiments [4].The saturation of the analysed absolute instability is provide by nonlinear effects among which one should mention the amplitude dependent stochastic damping of the IB wave or further decay of the weakly damped EB daughter wave into the EB mode of the toroidal cavity and IB wave. In both cases one may expect intensive interaction of the produced IB waves with the ion component and fast ion tail production. Investigation of the instability nonlinear saturation as well as description of the fast convective instability which takes place in the vicinity of the pump beam will be given in the further coming papers.

\section{Conclusion}

Summarizing we would like to stress the possible role of the low-threshold absolute PDI predicted in this paper in anomalous absorption of microwave power and, in particular, in fast ion production often observed in second harmonic ECRH in toroidal plasmas.

\section{Acknowledgements}

Work supported by RFBR grants 12-02-90003-Bel, 10-02-00887, NWO-RFBR Centre of Excellence on Fusion Physics and Technology (grant 047.018.002), the RAS Presidium program №12 and the RF government grant № 11.G34.31.0041

\section{References}

1. $\quad$ M. Porkolab and B.I. Cohen, Nucl. Fusion 28, 239 (1988)

2. B.I. Cohen, R.H. Cohen, W.M. Nevins and T. D. Rognlien, Rev. Mod. Phys. 63, 949 (1991)

3. A.G. Litvak, A.M. Sergeev, E.V. Suvorov et al., Phys. Fluids B 5, 4347 (1993)

4. V.F. Andreev, Yu.N. Dnestrovskij, M.V. Ossipenko et al., Plasma Phys. Control. Fusion 46, 319 (2004)

5. J.W. Oosterbeek, A. Burger, E. Westerhof et al., Rev. Sci. Instrum. 79, 093503 (2008) 
6. $\quad$ E. Westerhof, S.K. Nielsen, J.W. Oosterbeek et al., Phys. Rev. Lett. 103, 125001 (2009)

7. D. Rapisadra, B. Zurro, V. Tribaldos et al., Plasma Phys. Control. Fusion 49, 309 (2007)

8. A.N. Karpushov, B.P. Duval, T.P. Goodman et al., in Proceedings $33^{\text {rd }}$ EPS Conference on Plasma Phys. Rome, 19 - 23 June 2006 ECA vol. 30I, P-1.152 (2006)

9. E.Z. Gusakov, A.Yu. Popov, JETP Letters 91, 655 (2010)

10. M. Kantor, A.J.H. Donne, R. Jaspers et al., Plasma Phys. Control. Fusion 51, 055002 (2009)

11. E.Z. Gusakov, A.Yu. Popov, Phys. Rev. Lett. 105, 115003 (2010)

12. E.Z. Gusakov, A.Yu. Popov, Nucl. Fusion 51, 073028 (2011)

13. E.Z. Gusakov, A.Yu. Popov, JETP Letters 94, 277 (2011)

14. D.G. Swanson Plasma Waves (Boston: Academic Press) (1989)

15. A.D. Piliya, A.N. Saveliev, Plasma Phys. Control. Fusion 36, 2059 (1994)

16. V. P.Silin Parametric Action of High Power Radiation on Plasma [in Rus], Moscow (1973)

17. E.Z. Gusakov, V.I. Fedorov, Sov. J. Plasma Phys. 5, 463 (1979)

18. S. Coda, S. Alberti, P. Blachard et al., Nucl. Fusion 43, 1361 (2003) 